\title{
Shared Experiences
}

\author{
Mike Seymour \\ University of Sydney \\ Sydney, NSW, Australia \\ mike.seymour@sydney.edu.au
}

Dirk S. Hovorka

University of Sydney

Sydney, NSW, Australia

dirk.hovorka@sydney.edu.au

\section{Judy Kay}

University of Sydney

Sydney, NSW, Australia

Judy.kay@sydney.edu.au

\begin{abstract}
Disciplines often approach phenomena from different perspectives and with different research tools. We offer this example of our efforts to embrace the wider $\mathrm{CHI}$ values through the exploration of emotive digital humans deployed in HCI. We designed and conducted an HCI experiment with mixed methods. In building an infrastructure that benefits from the strengths of both AIS SIGHCI and ACM SIGCHI research communities, we chose an approach that could reveal undisclosed worlds, hard to see from just one perspective. As technology offers HCI digital humans, new combined shared approaches may be needed to gain insights, especially prior to their wide scale deployment. As bridging related disciplines have failed in the past, perhaps a new approach is needed, one of shared experiences, especially when exploring new technological phenomenon.
\end{abstract}

Keywords Human Computer Interface, research approach. 


\section{Introduction}

Humans have highly developed neural pathways for facial interpretation and yet we provide no emotionally rich 'face' for interactions with our computational tools or 'partners'. Humans already interact with a wide range of cognitive agents, for example Apple's Siri or Amazon's Alexa. The application of highly accurate digital faces may add to the effectiveness of these cognitive agents and also may be effective as avatars in VR and as actors in communication and learning forums.

We offer a research example of "facing the computer" applying realistic interactive digital people. Our research benefited from using the shared experiences of both IS HCI and IT CHI. This hybrid approach was also informed by AI techniques, now being applied by HCI researchers, and applications of machine learning being increasingly visible in the HCI literature (Grudin, 2009). We offer this multi-staged research program as an example of how to approach the issue, while leaving the specific research questions and results to various separate publications handling each of the specific research projects. We note that using methods from two different approaches is not necessarily new, but as shown here, it can be very informative.

The research program spanned a range of research including a Delphi study, elements of Design Science Research, qualitative and quantitative data gathering. The program continues today with new research building on this successful base.

To clarify labels, in this paper, we adopt the convention of stating that Computer Human Interfaces (CHI) belongs as a label to the community that researchs and publishes in the Information Technology (IT) discipline, for example the ACM SIGCHI community. This is a narrower focus than the Human Computer Interface (HCI), Information Systems (IS) community, that might publish in the AIS SIGHCI (Grudin, 2017). We shall adopt the one 'HCI' label convention to encapsulate the interface discussion contained in our research document.

\subsection{Facing the computer}

The "facing the computer" research program using realistic interactive digital people benefited from applying a hybrid approach of research methodologies. The program also benefited from the AI techniques available to $\mathrm{CHI}$ researchers and the applications of machine learning being increasingly visible in the HCI literature (Grudin, 2009). We simulated AI components in our experiments by using machine learning (ML) and deep learning (DL) to implement the research projects.

\subsubsection{Study background}

For the actual second study, following on from the Delphi research we used a field study to investigate whether users had different affinity, trustworthiness, and preferences for avatars with two levels of realism, one photo-realistic and one a cartoon caricature. We collected survey data and conducted oneon-one interviews with SIGGRAPH conference attendees who watched a live interview carried out utilising two avatars, either on a large screen 2D video display or via 3D VR headsets. 18 sessions were conducted over four days, with the same person animating the photo realistic avatar but with different individuals animating the caricature avatars. While the actual details of this study will be publicised elsewhere, in summary, the participants rated the photo-realistic avatar more trustworthy, had more affinity for it, and preferred it as a virtual agent. Participants who observed the interview through VR headsets had even stronger affinity for the photo-realistic avatar and stronger preferences for it as a virtual agent.

\subsection{Common language}

When we speak broadly of two worlds CHI and HCI, dichotomies are problematic, but there is more overlap than difference. Defining the two communities as representing IS and IT is both limiting and implies separation rather than commonality with differing perspectives. We found both groups cover much of the same intellectual ground, but with different terminology describing the same thing, or equally, a common term is used differently between them. For example, a 'process' from one perspective refers to a 'CPU process' but a 'business process' from the other. This gap is not new, Grudin (2017) notes several such instances. A successful 'implementation' may mean code in one world and introduction to an organisation in another. What IS might call 'systems', an IT community may refer to as 'applications'. 'Task Analysis' is an organisational decomposition of work for IS, and in CHI it is more likely to be a cognitive decomposition (Grudin, 2017). Similarly, what one group may refer to in their literature as 'Common Understanding', may be similar to (but not identical to) 'Community of Practice' from a 
different group. Such differences do not directly map to the literature of the CHI or the HCI communities, and within each of these HCI communities there are also different groups with diverse perspectives.

From their own web sites, ACM SGI's CHI focus is on interaction between one or more humans and one or more computational machines ("ACM SIGCHI Curricula for Human-Computer Interaction : 2. Definition and Overview of Human-Computer Interaction," n.d.), while the AIS HCI's focus is the IS community, emphasizing applications in business, managerial, organizational, and cultural contexts (Association for Information Systems., n.d.).

\subsection{Institutionalised division}

One of the principle reasons for the divided community, apart from language and terminology, has been the different academic engagement and communication strategies of each community. Those researchers identifying with the IT community are rewarded for publishing at conferences, while the IS community values journal publications. It was estimated, at the opening of the 2004 HICSS conference, that less than $15 \%$ of the work in CHI sponsored conference research reaches journal publication. Whereas IS conference organizers have estimated that $80 \%$ of research presented at a leading IS conference progressed to a journal 1 .

\subsection{A historical failure to bridge}

For many years, bridging the disciplines has failed. Even in an environment where interdisciplinary cooperation is encouraged, and there is a lack of literature speaking against such bridge building efforts, there is an absence and gap of successful cases to draw on. IS has broadly sort overarching theory and focused on hypothesis testing. IT has become atheoretical and moved to less-rigorous methods such as cognitive walkthroughs in an effort to gain a deeper understanding when context is critical and unfamiliar (Grudin, 2017).

a) This difference and gap has happened even as many of the same issues have been researched in each of the two communities.

b) Our enquiry benefited from both specific interface interaction and exploring broader business ethical and theoretical issues.

\subsection{Areas of interest and matters of concern}

While bridging two different worlds has failed historically, the term 'bridging' also suggests the problem is that there are two separate and different worlds. As mentioned, this is not representative as the areas of interest overlap greatly. But equally what is not needed is a transdisciplinary solution that would fully span both worlds. There are legitimate and important differences. The solution is not a simple spanning, combining or bridging of two worlds.

It is apparent that new areas of HCI, such as fully realistic digital humans, has created what Latour called (positively) 'matters of concern' (Latour, 2004). The research of digital humans has many varied groups interested, from educators to the military. We need a space where the participants can gather and research the topic, that is not bound by one discipline's strict approach.

We attempted to overcome the dichotomization, which is largely institutional, by suggesting not a new or combined discipline, nor even a multi-discipline approach. We suggest a new focus on complexity studies by shared experiences from two disciplines, drawing on strengths from both groups, but without labeling or specifically targeting one or the other.

\subsection{Facing the future}

Our prior work was to build an in situ 'digital human' and that led to this hybrid research approach. For example, when the digital human is driven by a real person (as in an avatar), is the avatar ascribed with the traits of the user controlling it? Or is it a separate entity even if it is known to be controlled by a human, in much the same way a puppet can appear to be separate from the puppeteer who voices it? Users may see the avatar either standing apart from the user or as a direct extension of the user. This research project benefited from both an engineering inclined perspective, as well as a philosophical view. We joked that our work has benefited from 'cutting edge code with existential phenomenology'.

\footnotetext{
${ }^{1}$ Nunamaker, J. Opening remarks of HICSS-38.
} 
It should also be noted that this area of human simulation is closely related to current resurgent discussions on Artificial Intelligence. It has been stated that AI and HCI are currently converging (Grudin, 2009). Due to the deployment of many waves of technology such as Intelligent systems, Enterprise-wide Decision Support Systems, Expert Systems, etc. there is a long-standing link between the HCI community and the AI business research focused communities. Recent developments such as realistic virtual human agents once again under scores this point. While our research used ML to deal with technical issues. It is worth noting, for research design reasons, we choose to separate the specific implementation of AI human emulation from our program of research. While super-intelligent computers are speculated about, we assume that they are not imminent. While people may andromorphise these interfaces, we see them as digital partners that only amplify human agency. They may appear 'Intelligent', but they do not possess actual intelligence.

We designed a complex and highly advanced program involving multiple digital humans at different levels of realism, engagement and modal interaction in a contextual research environment. We did this so we could explore the issues raised by our initial Delphi study conducted with 13 senior industry experts from around the world.

We are researching a world of human computer symbiosis where people and computers share tasks and work together to reach goals. This supportive role of computers has the devices typically receding into the background. We aimed to reduce novelty by using primarily informed participants in the research. This was deemed to be key as many of the technologies were brought together for the first time for this research using a global research team involving four different countries (USA, Serbia, UK, and Australia).

We aimed to provide actual significant interactions so as to make the interaction as valid as possible. By engaging the avatars through a variety of platforms (i.e. on screen, in person and in VR) we also explicitly aimed to eliminate interface inferred biases.

People may be aware that their appliances have computers at their operational core, from fridges to phones, but these devices are not referred to as 'computers' but rather as 'smart' devices. These devices could potentially use new digital human interfaces and hence draw the user's attention to the foreground. The interface becomes the focus of attention as the technology is personified and humanised.

\section{Opening the Black Box : $\mathrm{HCl}$}

There is a valid HCI interest in exploring how a user interface can recede into the background, as part of the User Experience. Attention is thus focused on the task, and the awareness of interface recedes so interaction becomes frictionless. Such research has explored ubiquitous smart rooms or nomadic mobile technology that is ever accessible, without drawing attention to itself. In the past, this has led to a notion of black boxing the technology (Orlikowski \& Iacono, 2001).

In contrast, a first-person experience of an emotional interactive expressive human face represents a shift in research focus. Manifesting a cognitive agent as an emotionally engaging and intuitively responsive digital human would deliberately gather the focus of attention. The subject would be responding to and anticipating a human-looking face, wanting to make eye-contact and reading microgestures of empathy. What is the interpretation of such an experience? This area of digital humans provides the opportunity to research HCI as interfaces both recede from view and simultaneously gather attention.

From this perspective, a cognitive agent could not be isolated as an interface to technology, as it cannot be black boxed as an arbitrary piece of technology that stands apart with an identifiable interface. Here echoes can be heard of Merleau-Ponty's thought experiment when he asks the reader to imagine a blind man navigating a city street with a cane. What is the relationship and limits between that cane and the man's perceptual apparatus? (Matthews, 2010). Philosophers such as Merleau-Ponty disputed the Cartesian view that the mind and the brain/body can be considered anything other than one nondecomposable whole (Matthews, 2010). Similarly, we felt the implications of the technology could not be disclosed by discussion or looking at the IT artifact in isolation. The experience of the interaction must take its part in the referential whole. The notion of a black box standing apart is not valid.

From the IS toolbox, we used the approach of a qualitative Delphi study and from IT, the notion of prototyping. Combined with further qualitative and quantitative research, this provided a rich and dense level of insight and understanding. 
Conversely, while exploring sense making of this new evolving technology we did so without looking back at past IS tools. TAM is the most widely cited research in the IS community (Davis, Bagozzi, \& Warshaw, 1989). In the IS community, the central issue addressed by this prescriptive theory is one built around perceived ease of use, and perceived usefulness. TAM exemplifies the long term organisational view of technology and a top down MIS focus. In the IT CHI community 'perception' is secondary to "user satisfaction". In the CHI world, discretionary technology is Adopted by users not Accepted. A TAM approach was not used in our research. TAM comes from an IS context of internal organisational use of computer systems. The technology was bounded by the business structure. We expect the deployment of digital humans to not be an internally controlled or deployed vehicle of HCI. The focus will not be on a niche audience lead by the CIOs and CTOs of businesses, but the external broader social media landscape with cloud computing and consumer applications. In this respect, possible 'adoption' was explored with more of a CHI mixed methods approach that embraced exploration over hypothesis testing of potential 'acceptance'. TAM is indicative of an approach of compartmentalizing technology into a black box.

Similarly, regarding IT's tools, we noted that the nature of our exploration of future digital human agents is the interaction and meaning constructed as part of that interaction. It is holistic and inseparable, and it seemed worthy of both theoretical and philosophical reflection. The agent is an intrinsic part of the holistic environment and the co-defined dynamic interaction, yet it can be argued that current CHI research requires less of a theory. The continued focus on conferences reflects the focus on small scale, less theoretical research. CHI has a tendency, in a highly changing, technologically shifting environment, to focus on impact on individuals in society. It reflects a fast-moving tech world, but it can also presuppose smaller ethical and societal discussions of implications.

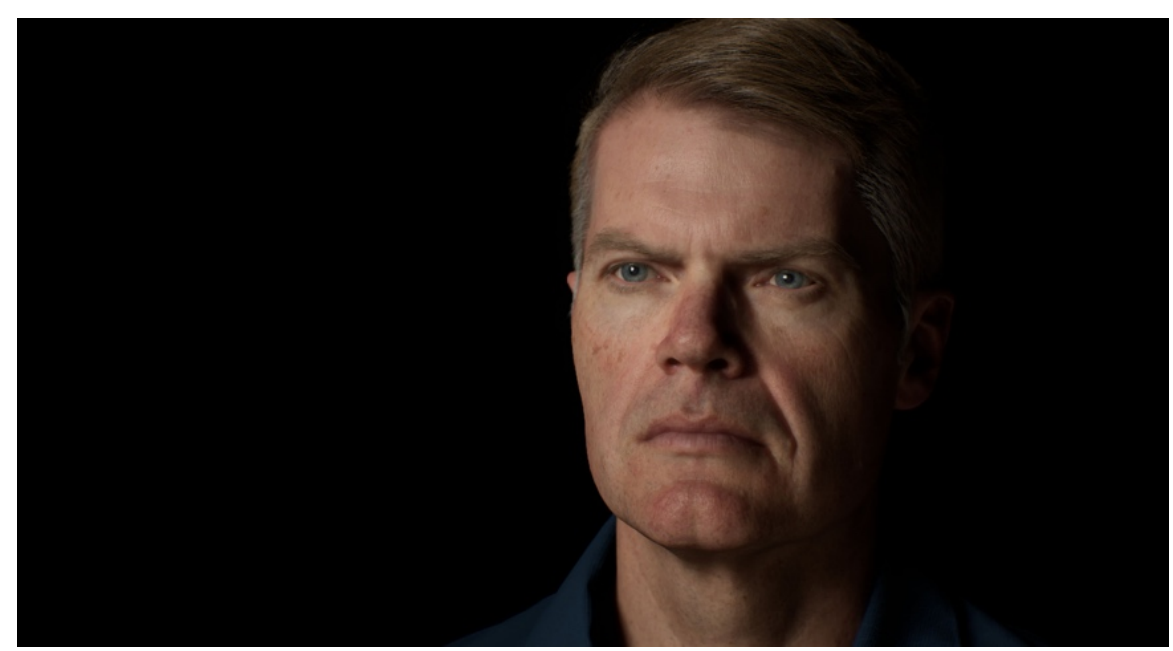

Figure 1: The realistic avatar could be rendered at 90 frames a second, interactively in Epic's UE4 engine, driven by markerless motion capture on stage, in real time and seen in Virtual Reality

\section{A Research Solution}

Our approach to exploring this area started with

- A multi-year Delphi study of leading industry and academic experts to define a vector for exploration.

- Building on those perspectives, we prototyped a real-time avatar system over an 18 month period (see figure 1, figure 2).

The impediment to adoption of digital humans in an HCI context has been strongly influenced by a 1970 s theory known as the Uncanny Valley (see figure 3).

Much research has examined how users respond to more realistic characters or avatars (Wang, Lilienfeld, \& Rochat, 2015). Of particular interest is the uncanny valley phenomenon, which refers to the effect that user affinity increases as realism increases, but drops sharply (hence a valley) as digital characters become almost but not quite human realistic (Mori, 1970; Mori, Kageki, \& MacDorman, 2012). Prior research has primarily examined user reactions to viewing static images or scripted prerecorded videos of avatars (Mathur \& Reichling, 2016; Wang et al., 2015). Hence, a key unanswered 
question is how interaction (i.e., unscripted communication) affects user perceptions, as such user engagement moves beyond simply the judgement of physical appearance to direct involvement with such characters. In this research, we focused our main investigation on interactions with avatars characters controlled by humans - to remove the variant issue of the realism of any artificial intelligence (AI) needed to enable non-human virtual agents to interact with humans in a meaningful way.

Our aim was to explore if we could cross the Uncanny Valley by producing a photo-real avatar which exhibited more 'affinity' with users. While 'affinity' is the best translation of the original Japanese word Shinwakan ( 親和感), we reasoned trust was conceptually more informative in HCI (Mori et al., 2012). Trust is a core value to both communities.

Our approach was to present both a cartoon like caricature avatar and a highly realistic avatar in conversation and allow users to witness this on screen, in person and in Virtual Reality. (See figure 5). We also explored if our photo-real avatar provided more affinity and if it did, if this correlated with more trustworthiness. This was triangulated with open ended qualitative interviews. The production of such highly responsive computer avatars was extremely complex requiring a global combined effort of several teams in four countries.

This research benefited from the infrastructure generated and the insight gained in the building. Countless decisions were required during the development phase, each of which, by the very asking, provide greater insight into what is and can be researched. For example, hand gesturing proved more significant than prior discussion had considered, this resulted in one character having articulated hands and one not (figure 4). Based on prior research into the algorithmic component of artificial agents (Sagar, Seymour, \& Henderson, 2016), our next stage of research was focused on the interpretation of what happens when a person engages with virtual humans. At the heart of the experience is the issue of agency and whose identity the observers believed they are experiencing, or how they view the embodiment.

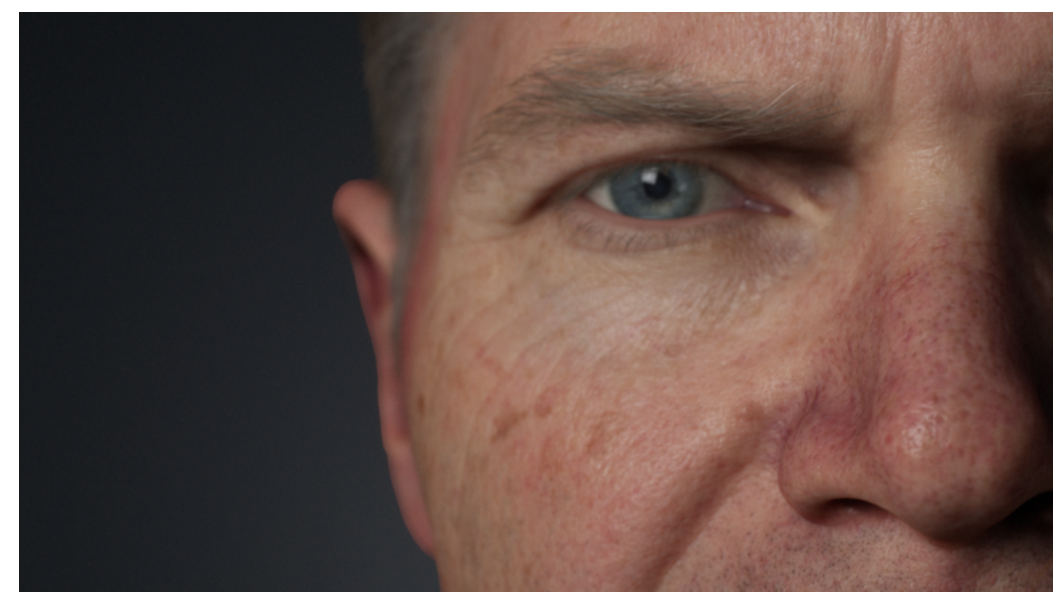

Figure 2: $A 3$ D rendered close up of the more realistic of the two avatars. See Figure 4 below for an example of the more 'cartoon' second avatar

In our experiment two people meet in VR. One meets with a cartoon avatar, the other with a highly realistic avatar. They are observed interacting by an audience in VR and on standard screens in real time. As both avatars represent experts, we examine how each was accepted. This research explores new areas of possible HCI so neither community's prior work, fully embraced the specific ethical issues we speculated about. Namely, issues surrounding trust and the role of avatars as stand-ins for ourselves in everyday life.

Our framing of the problem involves:

1. Defining the multiple phenomena including technical, attitudinal (acceptance) and phenomenological (trust and presence).

2. Measuring these phenomena of interest with mixed methods.

Generalizability of findings through the lens of existential presence. 


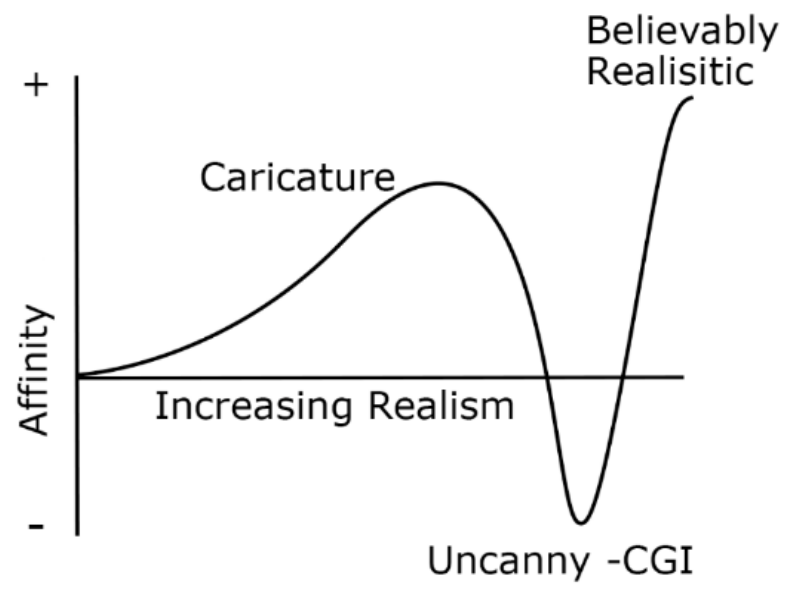

Figure 3: The Uncanny Valley theory refers to the effect that user affinity increases as character realism increases, but drops sharply as digital characters become almost but not quite human realistic. This is reversed again as the likeness becomes highly realistic. This non-linear effect has been cited as the reason for limited virtual character adoption.

\section{Blending of research experiences}

We learned that shared experiences from both IT and IS are a rich source of insight. It is vital that we explore the actual interaction of real people when presented with a new and novel experience enabled by technology. A key feature is that technology is not presented as technology with a distinct interface for the user to manipulate. The need to holistically explore the interaction and not see the interaction as a state machine model of separated users and black box technology, required the building of new technology based on expert opinion.

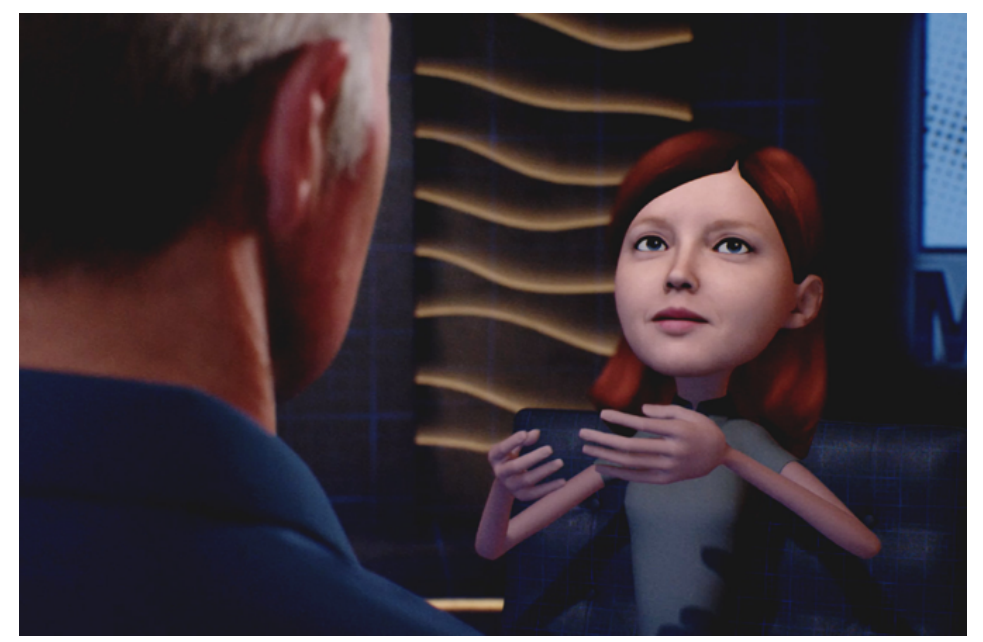

Figure 4: An example cartoon caricature as seen in the VR gear, note the second character's hands.

\section{Summary}

The research benefited from embracing perspectives from the practical lessons of building infrastructure and the philosophical nature of presence and experience theory. In so doing we believe valuable insights have been gained. A shared approach demonstrated a powerful way to explore new $\mathrm{HCl}$ technologies.

\subsection{Future Research}

The specific finding of improved trustworthiness, both qualitative and quantitative, are to be published separately in journals and at conferences. This reflects and acknowledges that there 
exists institutional dichotomization, which cannot be ignored. It is also true that the barriers of a common language can be time consuming. A shared understanding, within a discipline, does allow for validation of approaches and thinking. Tribalism, such as it is, aids understanding by commonality of language and approach.

Our decision to exclude an actual $\mathrm{Al}$ engine as the digital human driver during the avatar-human observed interactions was due to the research program constraints and the constructs under evaluation. But actual rather than emulated avatars without $\mathrm{Al}$ is an important future research opportunity. Such a digital human experiment would explore further the implications of broad Al to $\mathrm{HCl}$ adoption.

Looking to the future, building on this work from a very different perspective might be instructive. For example, exploring the nature of the research approach and language differences by outlining the $\mathrm{CHI} / \mathrm{HCl}$ research using the 'same' approach. In other words, outlining how to approach the same research questions(s) from both a strong IT Design Science Research approach and independently (from an equally rigorous) IS Design Science Research approach. There may be much to be learnt by examining how this topic could have been approached differently by a variety of different methodologies common yet different in each discipline. The side by side comparison of how each discipline would frame this problem may be informative in both the differences and the similarities.

\subsection{Shared Experiences}

While the stated focus of the different research communities may be different, there is much to be gained by embracing the benefits of the shared experiences when viewing new emerging $\mathrm{HCl}$ worlds, such as interactive virtual humans. AIS SIGHCl and ACM SIGCHI communities are different enough to be considered different disciplines, but they span much of the same space. By bringing their views and strengths together on innovative projects, we believe we can explore new ground, that neither perspective could explore as well independently.

\section{References}

ACM SIGCHI Curricula for Human-Computer Interaction : 2. Definition and Overview of HumanComputer Interaction. (n.d.). Retrieved March 10, 2018, from http://old.sigchi.org/cdg/cdg2.html

Association for Information Systems. (n.d.). AIS transactions on human-computer interaction. Association for Information Systems. Retrieved from http://aisel.aisnet.org/thci/

Davis, F. D., Bagozzi, R. P., \& Warshaw, P. R. (1989). User acceptance of computer technology: comparison of two theoretical models. Management Science, 35(8), 982-1003.

Grudin, J. (2009). AI and HCI: Two Fields Divided by a Common Focus. AI Magazine, 3o(4), 48. https://doi.org/10.1609/AIMAG.V30I4.2271

Grudin, J. (2017). From Tool to Partner: The Evolution of Human-Computer Interaction. Synthesis Lectures on Human-Centered Informatics, $10(1), \quad \mathrm{i}-183$. https://doi.org/10.2200/So0745ED1V01Y201612HCIo35

Latour, B. (2004). Why Has Critique Run out of Steam? From Matters of Fact to Matters of Concern. Critical Inquiry, 3O(2), 225-248. https://doi.org/10.1086/421123

Mathur, M. B., \& Reichling, D. B. (2016). Navigating a social world with robot partners: A quantitative cartography of the Uncanny Valley. Cognition, 146, 22-32. https://doi.org/10.1016/j.cognition.2015.09.008

Matthews, E. (2010). Maurice Merleau-Ponty: Phenomenology of perception. In Central Works of Philosophy Volume 4: The Twentieth Century: Moore to Popper (pp. 177-194). https://doi.org/10.1017/UPO9781844653614.011

Mori, M. (1970). The uncanny valley. Energy, 7, 33-35. Retrieved from http://ieeexplore.ieee.org/xpls/abs_all.jsp?arnumber $=6213238$

Mori, M., Kageki, N., \& MacDorman, K. F. (2012). The Uncanny Valley. IEEE Robotics \& Automation Magazine, (June), 98-100. 
Orlikowski, W. J., \& Iacono, S. (2001). Desperately seeking the 'IT'in IT research: A call to theorizing the IT artifact. Information Systems Research, 12(2), 121-134. https://doi.org/10.1287/isre.12.2.121.9700

Sagar, M., Seymour, M., \& Henderson, A. (2016). Creating connection with autonomous facial animation. Communications of the ACM, 59(12), 82-91. https://doi.org/10.1145/2950041

Wang, S., Lilienfeld, S. O., \& Rochat, P. (2015). The Uncanny Valley: Existence and Explanations. Review of General Psychology, 19(4), 393-407. https://doi.org/10.1037/gprooooo56

\section{Copyright}

Copyright: (C) 2018 authors. This is an open-access article distributed under the terms of the $\underline{\text { Creative }}$ Commons Attribution-NonCommercial 3.0 Australia License, which permits non-commercial use, distribution, and reproduction in any medium, provided the original author and ACIS are credited. 